\title{
The Pre-Schwarzian Norm Estimate for Analytic Concave Functions
}

\author{
Young Jae Sim and Oh Sang Kwon \\ Department of Mathematics, Kyungsung University, Busan 608-736, Republic of Korea \\ Correspondence should be addressed to Oh Sang Kwon; oskwon@ks.ac.kr
}

Received 6 February 2015; Revised 27 March 2015; Accepted 29 March 2015

Academic Editor: Harvinder S. Sidhu

Copyright (C) 2015 Y. J. Sim and O. S. Kwon. This is an open access article distributed under the Creative Commons Attribution License, which permits unrestricted use, distribution, and reproduction in any medium, provided the original work is properly cited.

Let $\mathbb{D}$ denote the open unit disk and let $\delta$ denote the class of normalized univalent functions which are analytic in $\mathbb{D}$. Let $\operatorname{Co}(\alpha)$ be the class of concave functions $f \in \mathcal{S}$, which have the condition that the opening angle of $f(\mathbb{D})$ at infinity is less than or equal to $\pi \alpha, \alpha \in(1,2]$. In this paper, we find a sufficient condition for the Gaussian hypergeometric functions to be in the class $\operatorname{Co}(\alpha)$. And we define a class $\operatorname{Co}(\alpha, A, B),(-1 \leq B<A \leq 1)$, which is a subclass of $\operatorname{Co}(\alpha)$ and we find the set of variabilities for the functional $\left(1-|z|^{2}\right)\left(f^{\prime \prime}(z) / f^{\prime}(z)\right)$ for $f \in \operatorname{Co}(\alpha, A, B)$. This gives sharp upper and lower estimates for the pre-Schwarzian norm of functions in $\operatorname{Co}(\alpha, A, B)$. We also give a characterization for functions in $\operatorname{Co}(\alpha, A, B)$ in terms of Hadamard product.

\section{Introduction}

Let $\mathscr{H}$ denote the class of functions analytic in the unit disk $\mathbb{D}=\{z \in \mathbb{C}:|z|<1\}$. We denote the class of locally univalent functions by $\mathscr{L} \mathscr{U}$. Let $\mathscr{A}$ denote the class of functions $f \in \mathscr{H}$ with normalization $f(0)=f^{\prime}(0)-1=0$ and let $\mathcal{S}$ be the class of functions in $\mathscr{A}$ that are univalent in $\mathbb{D}$. Also we define the subclass $\mathscr{K} \subset \mathcal{S}$ of convex functions whenever $f(\mathbb{D})$ is a convex domain.

A function $f: \mathbb{D} \rightarrow \mathbb{C}$ is said to belong to the family $\operatorname{Co}(\alpha)$ if $f$ satisfies the following conditions:

(i) $f$ is analytic in $\mathbb{D}$ with the standard normalization $f(0)=f^{\prime}(0)-1=0$. In addition, it satisfies $f(1)=\infty$.

(ii) $f$ maps $\mathbb{D}$ conformally onto a set whose complement with respect to $\mathbb{C}$ is convex.

(iii) The opening angle of $f(\mathbb{D})$ at $\infty$ is less than or equal to $\pi \alpha, \alpha \in(1,2]$.

The class $\operatorname{Co}(\alpha)$ is referred to as the class of concave univalent functions. We note that for $f \in \operatorname{Co}(\alpha), \alpha \in(1,2]$, the closed set $\mathbb{C} \backslash f(\mathbb{D})$ is convex and unbounded. We observe that $\mathrm{Co}(2)$ contains the classes $\operatorname{Co}(\alpha), \alpha \in(1,2]$.
Avkhadiev and Wirths [1] found the analytic characterization for functions in $\operatorname{Co}(\alpha), \alpha \in(1,2]: f \in \operatorname{Co}(\alpha)$ if and only if

$$
\operatorname{Re}\left\{\frac{2}{\alpha-1}\left\{\frac{(\alpha+1)}{2} \frac{1+z}{1-z}-1-\frac{z f^{\prime \prime}(z)}{f^{\prime}(z)}\right\}\right\}>0, \quad z \in \mathbb{D} \text {. }
$$

For $f \in \mathscr{L} \mathscr{U}$, the pre-Schwarzian derivative $T_{f}$ is defined by $T_{f}=f^{\prime \prime} / f^{\prime}$ and we define the norm of $T_{f}$ by

$$
\left\|T_{f}\right\|=\sup _{z \in \mathbb{D}}\left(1-|z|^{2}\right)\left|T_{f}(z)\right| .
$$

It is well known that $\left\|T_{f}\right\| \leq 6$ for $f \in \mathcal{S}$ and $\left\|T_{f}\right\| \leq 4$ for $f \epsilon$ $\mathscr{K}$. In [2], Bhowmik et al. obtained the estimate of the preSchwarzian norm for functions $f \in \operatorname{Co}(\alpha)$ as the following:

$$
4 \leq\left\|T_{f}\right\| \leq 2 \alpha+2, \quad f \in \operatorname{Co}(\alpha) .
$$

For more investigation of concave functions, we may refer to [3-7].

We say that $f$ is subordinate to $F$ in $\mathbb{D}$, written as $f<F$, if and only if $f(z)=F(w(z))$ for some Schwarz functions $w(z)$, $w(0)=0$, and $|w(z)|<1, z \in \mathbb{D}$. If $F(z)$ is univalent in $\mathbb{D}$, 
then the subordination $f \prec F$ is equivalent to $f(0)=F(0)$ and $f(\mathbb{D}) \subset F(\mathbb{D})$.

By using the subordination, we define a subclass of concave functions as follows.

Definition 1. Let $A$ and $B$ be real numbers such that $-1 \leq B<$ $A \leq 1$. The function $f \in \mathscr{A}$ belongs to the class $\operatorname{Co}(\alpha, A, B)$ if $f$ satisfies the following:

$$
\frac{2}{\alpha-1}\left\{\frac{(\alpha+1)}{2} \frac{1+z}{1-z}-1-\frac{z f^{\prime \prime}(z)}{f^{\prime}(z)}\right\} \prec \frac{1+A z}{1+B z} \quad(z \in \mathbb{D}) .
$$

Note that $\operatorname{Co}(\alpha, 1,-1) \equiv \operatorname{Co}(\alpha)$.

Let $a, b$, and $c$ be complex numbers with $c \neq 0,-1$, $-2, \ldots$. We define the Gaussian hypergeometric function ${ }_{2} F_{1}(a, b, c ; z)$ by

$$
{ }_{2} F_{1}(a, b, c ; z):=F(a, b, c ; z)=\sum_{k=0}^{\infty} \frac{(a)_{k}(b)_{k}}{(c)_{k}} \frac{z^{k}}{k !},
$$

where $(\gamma)_{k}$ is Pochhammer symbol defined, in terms of Gamma function $\Gamma$, by

$$
\begin{aligned}
(\gamma)_{k}:= & \frac{\Gamma(\gamma+k)}{\Gamma(\gamma)} \\
& = \begin{cases}1, & (k=0) \\
\gamma(\gamma+1) \cdots(\gamma+k-1) & (k \in \mathbb{N}) .\end{cases}
\end{aligned}
$$

We note that the Gaussian hypergeometric function $F$ satisfies the hypergeometric differential equation

$$
z(1-z) F^{\prime \prime}(z)+[c-(a+b+1) z] F^{\prime}(z)-a b F(z)=0 .
$$

The Gaussian hypergeometric function has been studied extensively by various authors [8-13]. In particular, univalency, close-to-convexity, starlikeness, convexity, and various other properties associated with these hypergeometric functions were investigated based on the conditions of $a, b$, and $c$ in [14-17].

If $f \in \mathscr{H}$ and $g \in \mathscr{H}$ given by

$$
f(z)=\sum_{n=0}^{\infty} a_{n} z^{n}, \quad g(z)=\sum_{n=0}^{\infty} b_{n} z^{n},
$$

then Hadamard product (or convolution) $f * g \in \mathscr{H}$ of $f$ and $g$ is defined (as usual) by

$$
(f * g)(z)=\sum_{n=0}^{\infty} a_{n} b_{n} z^{n} .
$$

In this paper, we find a sufficient condition for the Gaussian hypergeometric functions to be in the class $\operatorname{Co}(\alpha)$. And we find the set of variabilities for the functional (1 $\left.|z|^{2}\right) T_{f}(z)$ and as a consequence of this we derive upper and lower bounds for the pre-Schwarzian norm $\left\|T_{f}\right\|$, for functions $f$ in $\operatorname{Co}(\alpha, A, B)$. And we give a representation formula in terms of Hadamard product for functions in $\operatorname{Co}(\alpha, A, B)$.

\section{A Sufficient Condition for Functions to $\mathrm{Be}$ in $\mathrm{Co}(\alpha)$}

In this section, we investigate a sufficient condition for the Gaussian hypergeometric functions to be in the class $\operatorname{Co}(\alpha)$. The proof of our result in this section is based upon the following lemmas.

Lemma 2 (see [18], Miller and Mocanu, p. 35). Let $\Omega$ be a set in the complex plane $\mathbb{C}$ and let $b$ be a complex number such that $\operatorname{Re}(b)>0$. Suppose that the function $\psi: \mathbb{C}^{2} \times \mathbb{D} \rightarrow \mathbb{C}$ satisfies the condition

$$
\psi(i x, y ; z) \notin \Omega
$$

for all real $x, y \leq-|b-i x|^{2} /(2 \operatorname{Re}(b))$ and all $z \in \mathbb{D}$. If the function $p(z)$ defined by $p(z)=b+b_{1} z+b_{2} z^{2}+\cdots$ is analytic in $\mathbb{D}$ and if

$$
\psi\left(p(z), z p^{\prime}(z) ; z\right) \in \Omega
$$

then $\operatorname{Re}\{p(z)\}>0$ in $\mathbb{D}$.

Lemma 3 (see [18], Miller and Mocanu, p. 239). If $a, b$, and $c$ are real and satisfy

$$
-1 \leq b \leq c, \quad a \in[-2,0) \cup[c-1, c+1],
$$

then $F^{\prime}(a, b, c ; z) \neq 0$.

Theorem 4. Let $\alpha \in(1,2]$ and $a, b$, and $c$ be real and satisfy (12) and

$$
\begin{gathered}
\alpha+c-1>|-\alpha+a+b-1|, \\
\operatorname{Re}\{D(z) \overline{B(z)}\} \leq 0,
\end{gathered}
$$

in $\mathbb{D}$, where

$$
\begin{gathered}
B(z)=2(\alpha-1)(1-z)\{(-\alpha+a+b-1) z-(\alpha+c-1)\} \\
D(z)=\{(\alpha+3)(\alpha-2 a-2 b-1)+4(a+1)(b+1)\} z^{2} \\
+\{2(\alpha+3)(\alpha+c)-2(\alpha-1)(a+b+1) \\
\quad-4(a+1)(b+1)\} z+(\alpha-1)(\alpha-1+2 c) .
\end{gathered}
$$

Then,

$$
\frac{c}{a b}\{F(a, b, c ; z)-1\} \in \operatorname{Co}(\alpha) .
$$

Proof. Let

$$
f(z)=\frac{c}{a b}\{F(a, b, c ; z)-1\} .
$$

Then, $f$ satisfies $f(0)=0, f^{\prime}(0)=1$, and

$$
F(z):=F(a, b, c ; z)=\frac{a b}{c} f(z)+1 .
$$


From the hypergeometric differential equation (7), we have $z(1-z) f^{\prime \prime}(z)+[c-(a+b+1) z] f^{\prime}(z)-a b f(z)-c=0$.

From condition (12) and Lemma 3, we have

$$
f^{\prime}(z)=\frac{c}{a b} F^{\prime}(a, b, c ; z) \neq 0
$$

in $\mathbb{D}$. If we set

$$
p(z)=\frac{2}{\alpha-1}\left\{\frac{\alpha+1}{2} \frac{1+z}{1-z}-1-\frac{z f^{\prime \prime}(z)}{f^{\prime}(z)}\right\},
$$

then $p$ is analytic in $\mathbb{D}$ and satisfies $p(0)=1$. Furthermore, we have

$$
\begin{aligned}
& (\alpha-1)(1-z) f^{\prime}(z) p(z) \\
& =(\alpha+1)(1+z) f^{\prime}(z)-2(1-z) f^{\prime}(z) \\
& \quad-2 z(1-z) f^{\prime \prime}(z) .
\end{aligned}
$$

Hence, we get

$$
\begin{aligned}
& 2 z(1-z) f^{\prime \prime}(z) \\
& \quad=\{\alpha-1+(\alpha+3) z-(\alpha-1)(1-z) p(z)\} f^{\prime}(z)
\end{aligned}
$$

If we use this substitution in (19), then

$$
\begin{aligned}
& \{\alpha-1+(\alpha+3) z-(\alpha-1)(1-z) p(z)\} f^{\prime}(z) \\
& \quad+2\{c-(a+b+1) z\} f^{\prime}(z)-2 a b f(z)-2 c \\
& =0 .
\end{aligned}
$$

Differentiating (24), we have

$$
\begin{aligned}
& \left\{\alpha+3+(\alpha-1) p(z)-(\alpha-1)(1-z) p^{\prime}(z)\right\} f^{\prime}(z) \\
& \quad+\{\alpha-1+(\alpha+3) z-(\alpha-1)(1-z) p(z)\} f^{\prime \prime}(z) \\
& \quad-2(a+b+1) f^{\prime}(z)+2\{c-(a+b+1) z\} f^{\prime \prime}(z) \\
& \quad-2 a b f^{\prime}(z)=0
\end{aligned}
$$

Substituting (23) in (25), we have

$$
\begin{aligned}
2\{\alpha & \left.+3+(\alpha-1) p(z)-(\alpha-1)(1-z) p^{\prime}(z)\right\} z(1-z) \\
& +\{\alpha-1+(\alpha+3) z-(\alpha-1)(1-z) p(z)\}^{2} \\
& -4(a+b+1) z(1-z)+2\{c-(a+b+1) z\} \\
& \cdot\{\alpha-1+(\alpha+3) z-(\alpha-1)(1-z) p(z)\} \\
& -4 a b z(1-z)=0 .
\end{aligned}
$$

And this equation leads us to the following first order differential equation:

$$
A(z) p^{2}(z)+B(z) p(z)+C(z) z p^{\prime}(z)+D(z)=0,
$$

where

$$
\begin{gathered}
A(z)=(\alpha-1)^{2}(1-z)^{2}, \\
B(z)=2(\alpha-1)(1-z)\{(-\alpha+a+b-1) z-(\alpha+c-1)\} \\
C(z)=-2(\alpha-1)(1-z)^{2}, \\
D(z)=\{(\alpha+3)(\alpha-2 a-2 b-1)+4(a+1)(b+1)\} z^{2} \\
+\{2(\alpha+3)(\alpha+c)-2(\alpha-1)(a+b+1) \\
-4(a+1)(b+1)\} z+(\alpha-1)(\alpha-1+2 c) .
\end{gathered}
$$

Since inequality (13) implies $B(z) \neq 0$ in $\mathbb{D}$, we can rewrite (27) in the form

$$
J(z) p^{2}+p(z)+K(z) z p^{\prime}(z)+L(z)=0,
$$

where

$$
J(z)=\frac{A(z)}{B(z)}, \quad K(z)=\frac{C(z)}{B(z)}, \quad L(z)=\frac{D(z)}{B(z)}
$$

A bilinear transformation $w(z)=(1+A z) /(1+B z)$, with $-1 \leq$ $A \leq 1$ and $-1 \leq B \leq 1$, has the property that $\operatorname{Re}\{w(z)\}>0$, for $z \in \mathbb{D}$. From the condition $\alpha \in(1,2]$ and (13), we have

$$
\begin{aligned}
& \operatorname{Re}\{K(z)\} \\
& =\frac{1}{\alpha+c-1} \operatorname{Re}\left\{\frac{1-z}{1+((\alpha-a-b+1) /(\alpha+c-1)) z}\right\}>0
\end{aligned}
$$

in $\mathbb{D}$ and

$$
\begin{aligned}
& \operatorname{Re}\left\{J(z)+\frac{1}{2} K(z)\right\} \\
& \quad=\frac{2-\alpha}{2(\alpha+c-1)} \operatorname{Re}\left\{\frac{1-z}{1+((\alpha-a-b+1) /(\alpha+c-1)) z}\right\} \\
& \quad \geq 0
\end{aligned}
$$

in $\mathbb{D}$. Now, we let $\Omega=\{0\}$ and define a function $\psi: \mathbb{C}^{2} \times \mathbb{D} \rightarrow$ $\mathbb{C}$ by

$$
\psi(r, s ; z)=J(z) r^{2}+r+K(z) s+L(z)
$$

Then, (27) becomes

$$
\psi\left(p(z), z p^{\prime}(z) ; z\right) \in \Omega
$$


By using (14), (31), and (32), we obtain

$$
\begin{aligned}
\operatorname{Re} & \{\psi(i \rho, \sigma ; z)\} \\
& =-\operatorname{Re}\{J(z)\} \rho^{2}+\operatorname{Re}\{K(z)\} \sigma+\operatorname{Re}\{L(z)\} \\
& \leq-\left(\operatorname{Re}\left\{J(z)+\frac{1}{2} K(z)\right\}\right) \rho^{2}-\frac{1}{2} \operatorname{Re}\{K(z)\}+\operatorname{Re}\{L(z)\} \\
& <\operatorname{Re}\{L(z)\} \\
& =\frac{\operatorname{Re}\{D(z) \overline{B(z)}\}}{|B(z)|^{2}} \\
& \leq 0
\end{aligned}
$$

for all $z \in \mathbb{D}$ and all real $\rho$, $\sigma$ with $\sigma \leq-\left(1+\rho^{2}\right) / 2$. By Lemma 2, we have $\operatorname{Re}\{p(z)\}>0$ in $\mathbb{D}$, which shows that $f \in \operatorname{Co}(\alpha)$.

Example 5. If we take $\alpha=2, a=3, b=1$, and $c=2$, then we can easily check that conditions (12) and (13) are satisfied. Furthermore,

$$
\operatorname{Re}\left\{\varphi_{1}(z)\right\} \leq 0 \quad(z \in \mathbb{D}),
$$

where

$$
\varphi_{1}(z)=D(z) \overline{B(z)}=2 \overline{(1-z)(-3+z)}\left(5-2 z-3 z^{2}\right)
$$

(see Figure 1). Hence,

$$
f_{1}(z)=\frac{2}{3} F(3,1,2 ; z)=\frac{2}{3}\left(-1+\frac{1-z / 2}{(1-z)^{2}}\right)
$$

belongs to $\operatorname{Co}(2)$, as shown in Figure 2.

Example 6. If we take $\alpha=3 / 2, a=3 / 2, b=1$, and $c=1$, then we can easily check that conditions (12) and (13) are satisfied. Furthermore,

$$
\operatorname{Re}\left\{\varphi_{2}(z)\right\} \leq 0 \quad(z \in \mathbb{D})
$$

where

$$
\varphi_{2}(z)=D(z) \overline{B(z)}=\frac{3}{8}(\overline{1-z})\left(z^{2}+4 z-5\right)
$$

(see Figure 3). Hence,

$$
f_{2}(z)=\frac{2}{3} F\left(\frac{3}{2}, 1,1 ; z\right)=\frac{2}{3}\left(-1+\frac{1-z / 2}{(1-z)^{3 / 2}}\right)
$$

belongs to $\operatorname{Co}(3 / 2)$, as shown in Figure 4 .

\section{The Pre-Schwarzian Norm Estimate for Functions in $\operatorname{Co}(\alpha, A, B)$}

Now, for $f \in \operatorname{Co}(\alpha, A, B)$, we find the exact set of variabilities for the functional $\left(1-|z|^{2}\right) T_{f}(z)$, which gives both sharp upper and lower bounds for the pre-Schwarzian norm $\left\|T_{f}\right\|$.

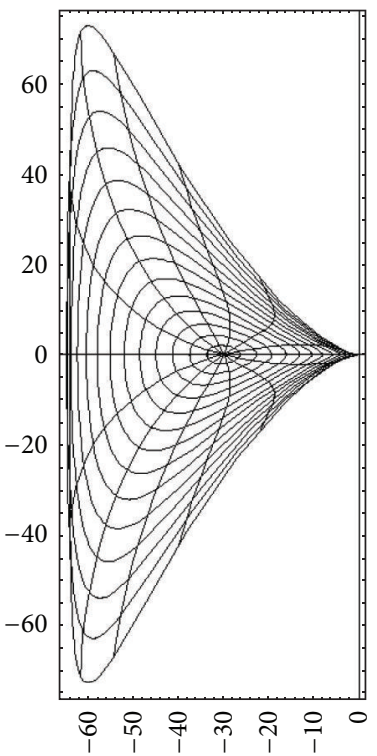

Figure 1: The image of $\varphi_{1}(z)$.

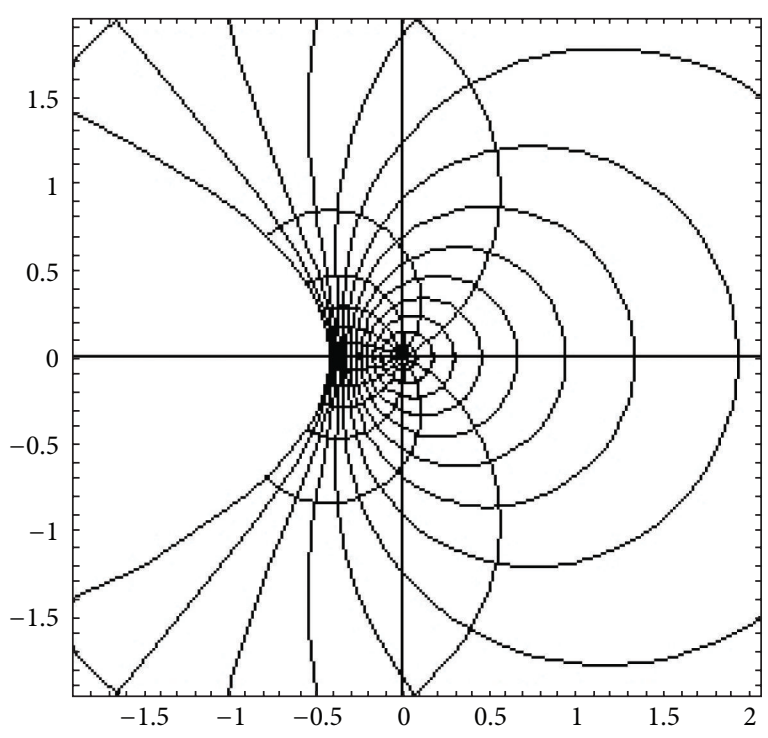

Figure 2: The image of $f_{1}(z)$.

Theorem 7. Let $\alpha \in(1,2]$ and $-1 \leq B<A \leq 1$ be fixed and let $f \in \operatorname{Co}(\alpha, A, B)$. Then, the set of variabilities of the functional $\left(1-|z|^{2}\right) T_{f}(z)$ is the closed disk with center

$$
\left(\alpha+1-\frac{B}{2}(B-A)(\alpha-1)\right) \bar{z}+(\alpha+1) \frac{1-\bar{z}}{1-z}
$$

and radius

$$
\frac{1}{2}(A-B)(\alpha-1) .
$$

The points on the boundary of this disk are attained if and only if $f$ is one of the functions $g_{\theta}$, where

$$
g_{\theta}(z)=\int_{0}^{z}\left(1+B e^{i \theta} t\right)^{(B-A)(\alpha-1) /(2 B)}(1-t)^{-(1+\alpha)} d t .
$$




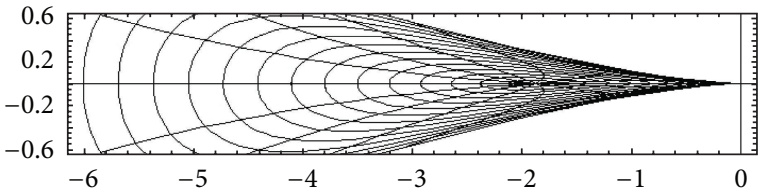

Figure 3: The image of $\varphi_{2}(z)$.

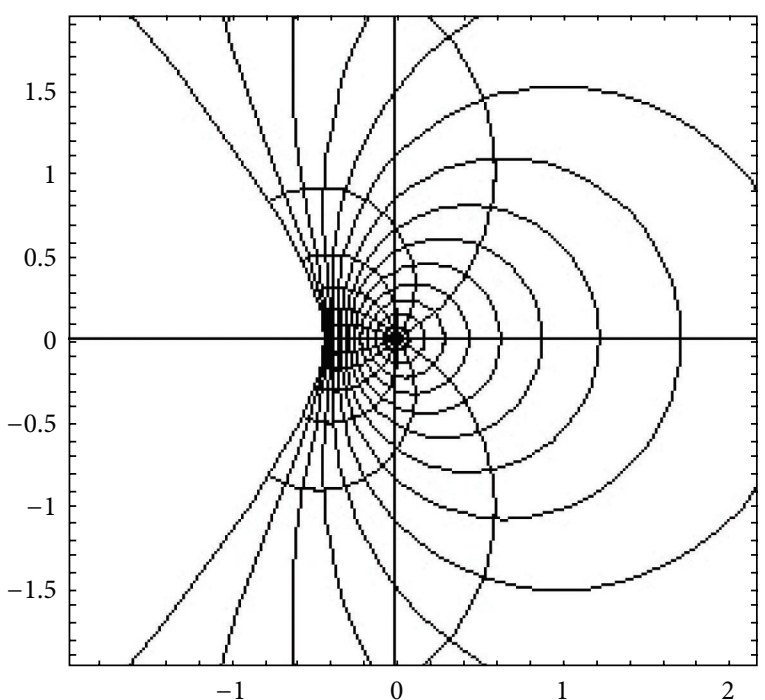

FIGURE 4: The image of $f_{2}(z)$.

Proof. We use the characterization (4) for functions in $\operatorname{Co}(\alpha$, $A, B)$ and the representation

$$
P_{f}(z)=\frac{1+A z w(z)}{1+B z w(z)}
$$

where $w: \mathbb{D} \rightarrow \overline{\mathbb{D}}$ is a unimodular bounded analytic function. It follows that

$$
\begin{aligned}
T_{f}(z)= & (2(\alpha+1)+(B-A)(\alpha-1) w(z) \\
& +((A+B) \alpha+3 B-A) z w(z)) \\
& \cdot(2(1-z)(1+B z w(z)))^{-1} .
\end{aligned}
$$

By a routine computation, one recognizes that

$$
\begin{aligned}
& \left(1-|z|^{2}\right) T_{f}(z)-\left(\alpha+1-\frac{B}{2}(B-A)(\alpha-1)\right) \bar{z} \\
& -(\alpha+1) \frac{1-\bar{z}}{1-z}=\frac{1}{2}(B-A)(\alpha-1) \frac{B \bar{z}+w(z)}{1+B z w(z)} .
\end{aligned}
$$

Hence, the condition $|w(z)| \leq 1$ is equivalent to

$$
\begin{gathered}
\mid\left(1-|z|^{2}\right) T_{f}(z)-\left(\alpha+1-\frac{B}{2}(B-A)(\alpha-1)\right) \bar{z} \\
-(\alpha+1) \frac{1-\bar{z}}{1-z} \mid \leq \frac{1}{2}(A-B)(\alpha-1) .
\end{gathered}
$$

This proves the first part of the assertion in the theorem. The second part follows from the fact that $|w(z)|=1$ if and only if $w(z) \equiv e^{i \theta}, \theta \in[0,2 \pi]$, and that the solution of the differential equation (4) in this case is given by $f(z)=g_{\theta}(z)$. The relation between boundary points of the above circle and the extremal function becomes clear from the identity

$$
\begin{gathered}
\left(1-|z|^{2}\right) T_{g_{\theta}}(z)-\left(\alpha+1-\frac{B}{2}(B-A)(\alpha-1)\right) \bar{z} \\
-(\alpha+1) \frac{1-\bar{z}}{1-z}=\frac{1}{2}(B-A)(\alpha-1) \frac{B \bar{z}+e^{i \theta}}{1+B e^{i \theta} z} .
\end{gathered}
$$

This completes the proof of the theorem.

Remark 8. If we put $A=1$ and $B=-1$ in Theorem 7 , then we can obtain the result in Bhowmik et al. ([2], Theorem 2.5).

From the inequality (48), we can have the following corollary.

Corollary 9. Let $f \in \operatorname{Co}(\alpha, A, B), \alpha \in[1,2]$ and $-1 \leq B<$ $A \leq 1$. Then,

$$
\begin{aligned}
& -\frac{1}{2}(1-B)(A-B)(\alpha-1)+2 \alpha+2 \\
& \quad \leq\left\|T_{f}\right\| \leq \frac{1}{2}(1+B)(A-B)(\alpha-1)+2 \alpha+2 .
\end{aligned}
$$

The equality holds in lower estimate for the function $g_{0}$ and in upper estimate for the function $g_{\pi}$ which are described in Theorem 7.

As a consequence of Theorem 7 , we can obtain a distortion theorem for the functions in $\operatorname{Co}(\alpha, A, B)$.

Theorem 10. Let $\alpha \in(1,2]$ and $-1 \leq B<A \leq 1$. Then, for each $f \in \operatorname{Co}(\alpha, A, B)$, one has

$$
\begin{aligned}
& \frac{(1-r)^{(1 / 4)(1-B)(A-B)(\alpha-1)}}{(1+r)^{(1 / 4)(1+B)(A-B)(\alpha-1)+(\alpha+1)}} \\
& \quad \leq\left|f^{\prime}(z)\right| \leq \frac{(1+r)^{(1 / 4)(1-B)(A-B)(\alpha-1)}}{(1-r)^{(1 / 4)(1+B)(A-B)(\alpha-1)+(\alpha+1)}}
\end{aligned}
$$

with $|z|=r<1$. For each $z \in \mathbb{D}, z \neq 0$, equality occurs if and only if $f=g_{\theta}$, where $\theta \in[0,2 \pi)$.

Remark 11. If we put $A=1$ and $B=-1$ in Theorem 10, then we can obtain the distortion theorem for the functions in $\operatorname{Co}(\alpha)$ which is a result from Bhowmik et al. ([2], Theorem 2.8).

Finally, we present a characterization for functions in the class $\operatorname{Co}(\alpha, A, B)$, in view of the Hadamard product. 
Theorem 12. Let $1<\alpha \leq 2$ and $-1 \leq B<A \leq 1$. Then, $f \in \operatorname{Co}(\alpha, A, B)$ if and only if

$$
\begin{aligned}
& \frac{1}{z}[f(z) *(((-A+B+\alpha A-\alpha B) z \\
& \left.+(-4 x+(A+3 B-\alpha A+\alpha B)) z^{2}\right) \\
& \left.\left.\cdot\left((1-z)^{3}\right)^{-1}\right)\right] \\
& +[f(z) *(((2 \alpha+2) x+(A-3 B-\alpha A-\alpha B)) z \\
& \left.+((2-2 \alpha) x+(-A-B+\alpha A+\alpha B)) z^{2}\right) \\
& \left.\left.\cdot\left((1-z)^{3}\right)^{-1}\right)\right] \neq 0
\end{aligned}
$$

for all $|z|<1$ and for all $x$ with $|x|=1$. Equivalently, this holds if and only if

$$
\sum_{n=0}^{\infty} E_{n} z^{n} \neq 0 \quad(z \in \mathbb{D},|x|=1),
$$

where

$$
\begin{gathered}
E_{0}=-\frac{1}{2}(A-B-\alpha A+\alpha B) \\
E_{n}=\left\{x-\frac{1}{2}(A+B-\alpha A+\alpha B)\right. \\
+(B-x)(n+1)\}(n+1) a_{n+1} \\
+\left\{\alpha x-\frac{1}{2}(-A+B+\alpha A+\alpha B)\right. \\
\quad-(-x+B) n\} n a_{n} \quad(n \in \mathbb{N})
\end{gathered}
$$

with

$$
f(z)=\sum_{n=1}^{\infty} a_{n} z^{n} \quad\left(a_{1}=1\right) .
$$

Remark 13. If we put $A=1$ and $B=-1$ in Theorem 12, then we can obtain the convolution result for the functions in $\operatorname{Co}(\alpha)$ which is a result from Bhowmik et al. ([2], Theorem 3.1).

\section{Conflict of Interests}

The authors declare that they have no competing interests.

\section{References}

[1] F. G. Avkhadiev and K.-J. Wirths, "Concave schlicht functions with bounded opening angle at infinity," Lobachevskii Journal of Mathematics, vol. 17, pp. 3-10, 2005.

[2] B. Bhowmik, S. Ponnusamy, and K.-J. Wirths, "Characterization and the pre-Schwarzian norm estimate for concave univalent functions," Monatshefte für Mathematik, vol. 161, no. 1, pp. 5975, 2010.
[3] F. G. Avkhadiev, C. Pommerenke, and K.-J. Wirths, "Sharp inequalities for the coefficients of concave schlicht functions," Commentarii Mathematici Helvetici, vol. 81, no. 4, pp. 801-807, 2006.

[4] F. G. Avkhadiev and K.-J. Wirths, "Convex holes produce lower bounds for coefficients," Complex Variables: Theory and Application, vol. 47, no. 7, pp. 553-563, 2002.

[5] B. Bhowmik, S. Ponnusamy, and K.-J. Wirths, "On the FeketeSzegö problem for concave univalent functions," Journal of Mathematical Analysis and Applications, vol. 373, no. 2, pp. 432438, 2011.

[6] L. Cruz and Ch. Pommerenke, "On concave univalent functions," Complex Variables and Elliptic Equations, vol. 52, no. 2-3, pp. 153-159, 2007.

[7] K.-J. Wirths, "Julia's lemma and concave schlicht functions," Quaestiones Mathematicae, vol. 28, no. 1, pp. 95-103, 2005.

[8] B. C. Carlson and D. B. Shaffer, "Starlike and prestarlike hypergeometric functions," SIAM Journal on Mathematical Analysis, vol. 15, no. 4, pp. 737-745, 1984.

[9] E. P. Merkes and W. T. Scott, "Starlike hypergeometric functions," Proceedings of the American Mathematical Society, vol. 12, pp. 885-888, 1961.

[10] S. Ponnusamy, "Hypergeometric transforms of functions with derivative in a half plane," Journal of Computational and Applied Mathematics, vol. 96, no. 1, pp. 35-49, 1998.

[11] S. Ponnusamy, "Starlikeness properties for convolutions involving hypergeometric series," Annales Universitatis Mariae CurieSkłodowska Sectio A, vol. 52, pp. 1-16, 1998.

[12] H. Silverman, "Starlike and convexity properties for hypergeometric functions," Journal of Mathematical Analysis and Applications, vol. 172, no. 2, pp. 574-581, 1993.

[13] A. Swaminathan, "Hypergeometric functions in the parabolic domain," Tamsui Oxford Journal of Mathematical Sciences, vol. 20, no. 1, pp. 1-16, 2004.

[14] Y. C. Kim and S. Ponnusamy, "Sufficiency for Gaussian hypergeometric functions to be uniformly convex," International Journal of Mathematics and Mathematical Sciences, vol. 22, no. 4, pp. 765-773, 1999.

[15] S. Ponnusamy, "Close-to-convexity properties of Gaussian hypergeometric functions," Journal of Computational and Applied Mathematics, vol. 88, no. 2, pp. 327-337, 1998.

[16] S. Ponnusamy and M. Vuorinen, "Univalence and convexity properties for Gaussian hypergeometric functions," Rocky Mountain Journal of Mathematics, vol. 31, no. 1, pp. 327-353, 2001.

[17] S. Ruscheweyh and V. Singh, "On the order of starlikeness of hypergeometric functions," Journal of Mathematical Analysis and Applications, vol. 113, no. 1, pp. 1-11, 1986.

[18] S. S. Miller and P. T. Mocanu, Differential Subordinations: Theory and Applications, Marcel Dekker, New York, NY, USA, 2000. 


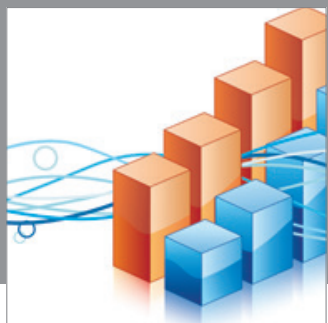

Advances in

Operations Research

mansans

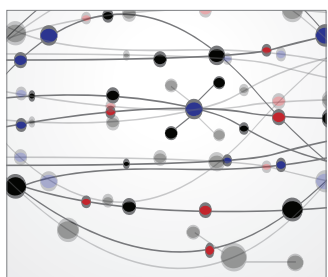

The Scientific World Journal
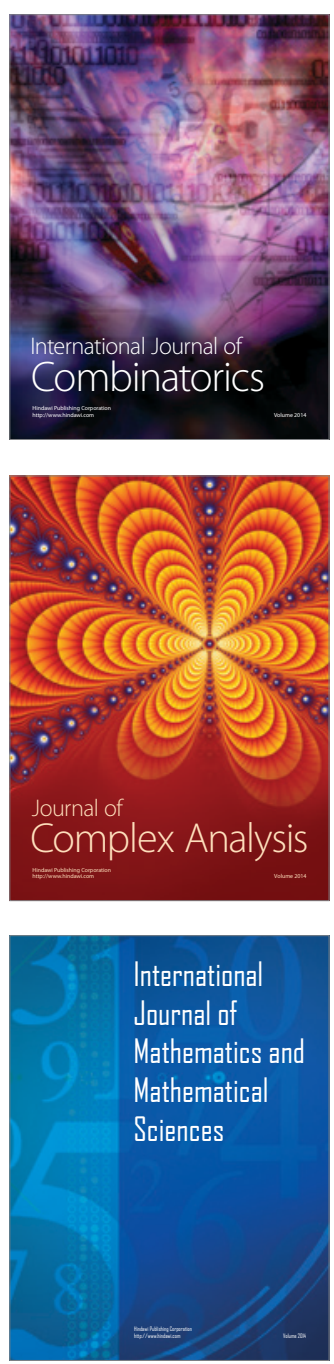
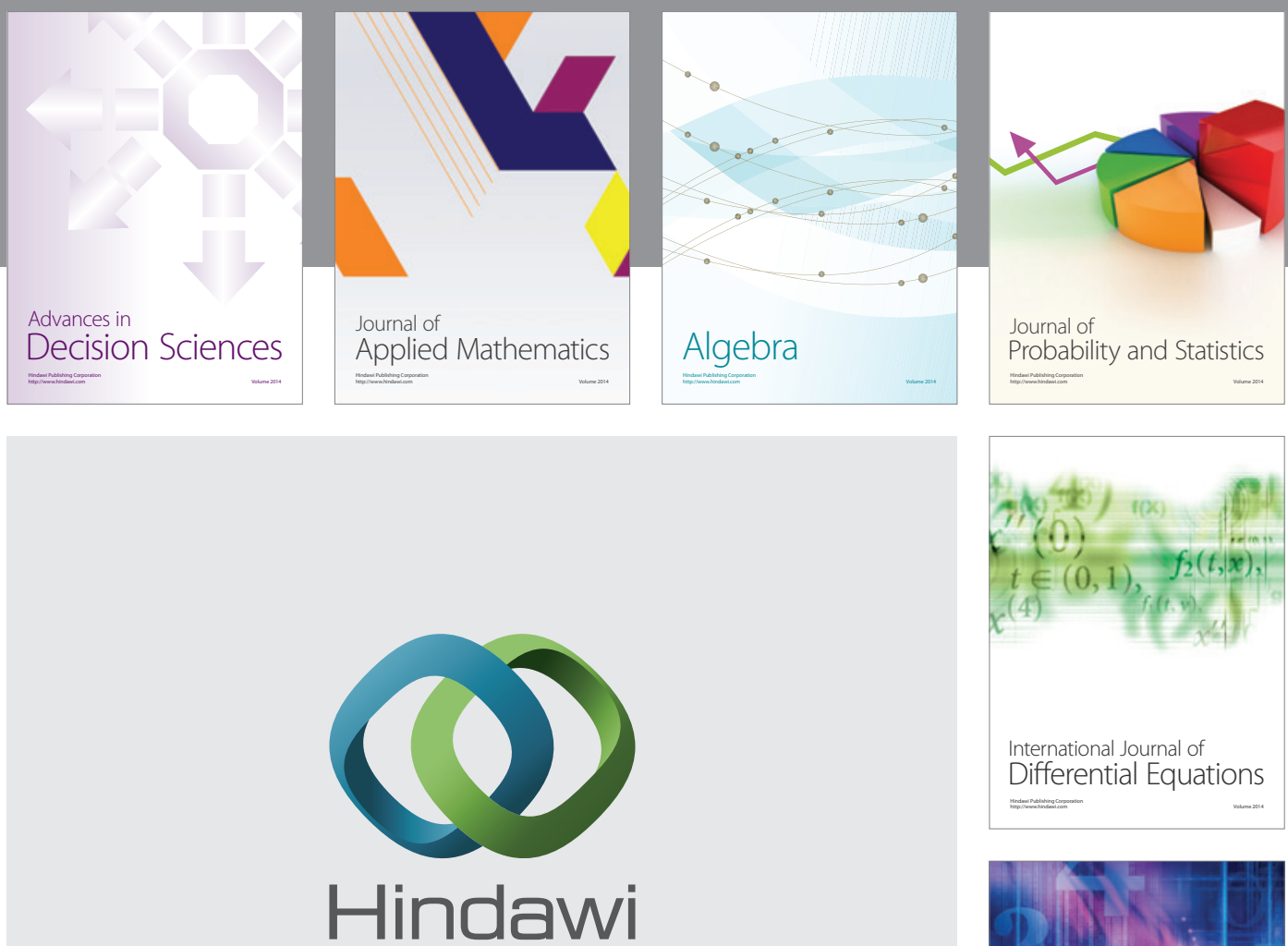

Submit your manuscripts at http://www.hindawi.com
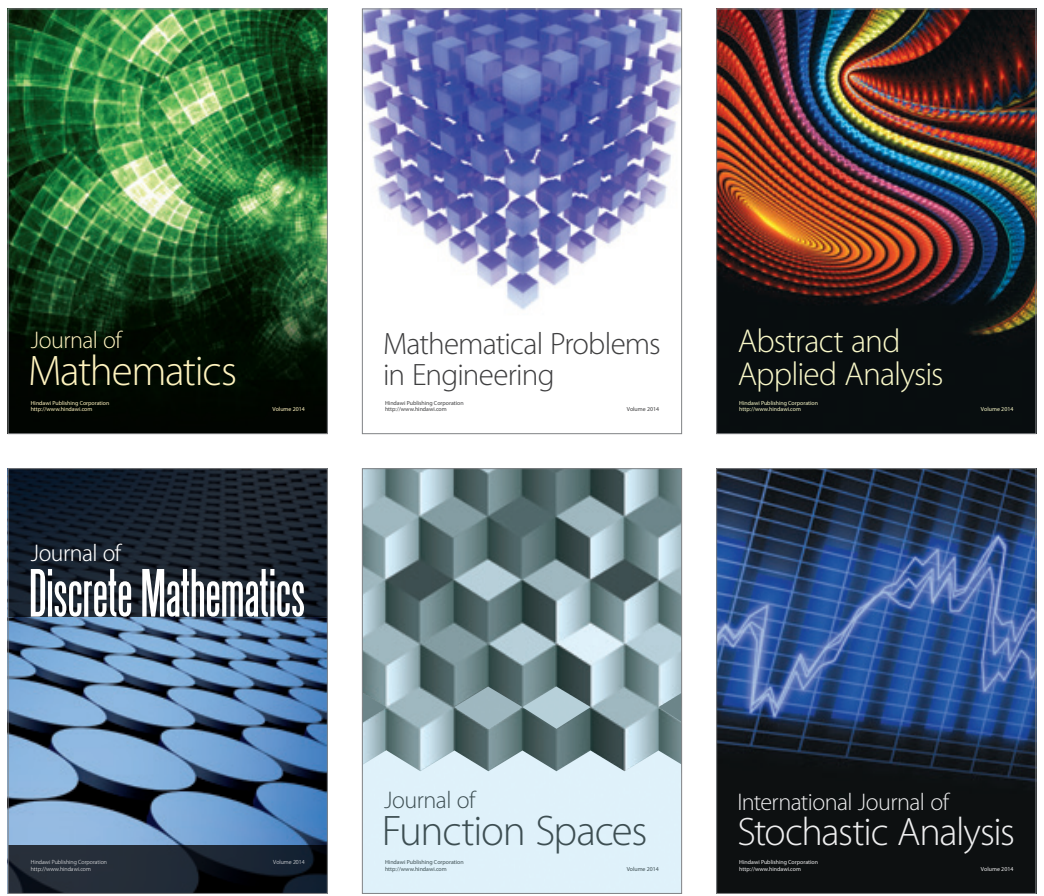

Journal of

Function Spaces

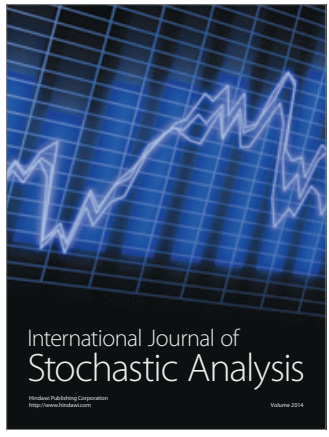

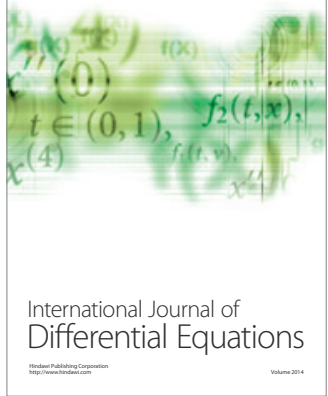
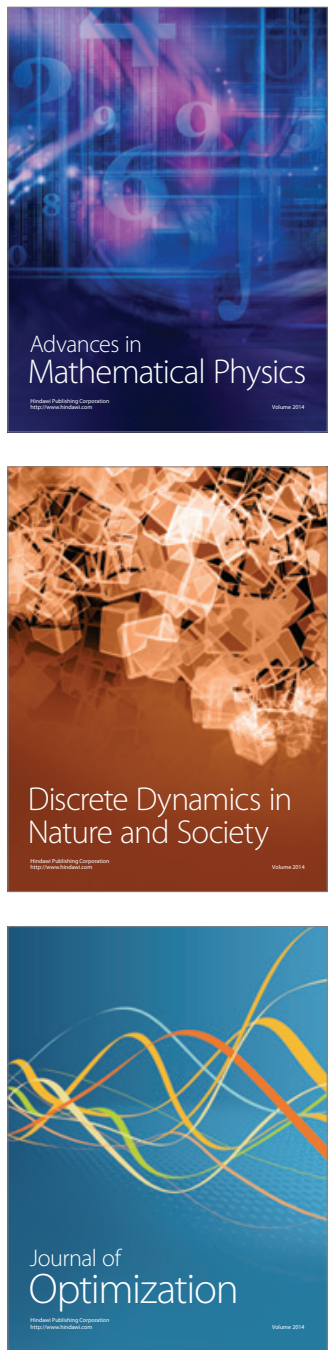